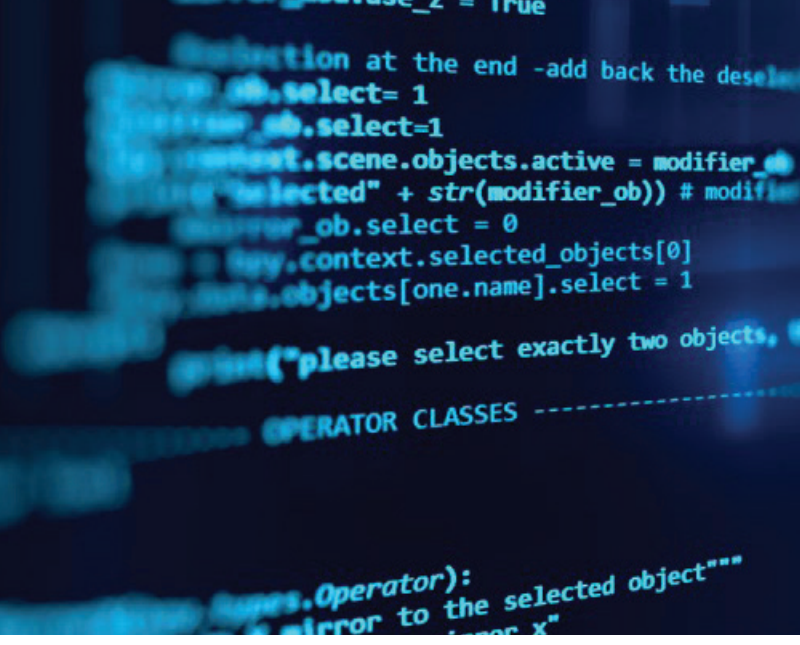

\title{
Modelo automatizado de procesos mediante el uso de herramientas de software business Process management, en gestión ambiental vial
}

\author{
Model automated processes by the use of business software tools process management, \\ environmental management road.
}

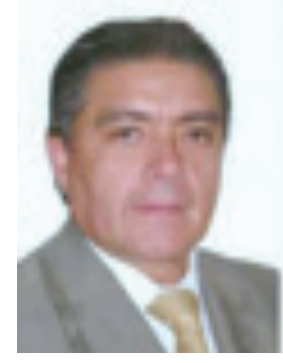

Ing. Patricio Ibarra Munizaga.

Consultor privado, Ingeniero Químico, Universidad Central del Ecuador; Magíster en Informática-Mención Redes (Cum Laude), Escuela Politécnica Nacional-Universidad Andina Simón Bolívar; Oracle Máster Oracle University / ibarra_patricio@yahoo.es

Resumen

En el presente trabajo, se analiza la importancia e interés del uso y aplicación de un Modelo Automatizado de Gestión por Procesos con herramientas de Software Business Process Management (BPM), ( BPMN/2.0.2/PDF/, 2013), a ser aplicado en la Gestión Ambiental Vial, especialmente adaptado a la Fase Constructiva; para el efecto, se hace un recorrido mediante un Flujo de Procesos, en conformidad a la Metodología actualmente aceptada; y sobre la base de los Procesos y Productos señalados en esta Estructura, se modelan los Procesos y Subprocesos de Gestión Ambiental. Una vez realizada esta actividad, se implementa el modelo con la herramienta BPM. Empleamos la herramienta Bizagi Modeler versión 10.4. Concluimos acerca de la utilidad de la aplicación para caracterizar el Modelo de Gestión por Procesos en el área Ambiental Vial.

Palabras clave: modelo automatizado; gestión por procesos; bpm; gestión ambiental, modelador bizagi

\section{Abstract}

This work discusses the importance and concern about the use and application of a computerized model of management by processes with tools of Software Business Process Management (BPM), (BPMN/2.0.2/PDF/, 2013) to be applied in the road environmental management, particularly adapted to the construction phase. For the effect, is a tour through a flow of processes, in accordance with currently accepted methodology; and based on processes and products mentioned in this structure, processes and threads of environmental management model. Once this activity, implements the model with the BPM tool. We use the tool Bizagi Modeler version 10.4. We conclude about the usefulness of the application to characterize the model of management by processes in the environmental Road area.

Keywords: computerized model; process management; bpm; environmental management; bizagi modeler 


\section{Introducción}

Una de las actividades que genera mayor impacto ambiental dentro de las obrashumanas, correspondealasconstrucciones viales y su mantenimiento que, por su importancia a nivel local y mundial, deben someterse a procesos permanentes de innovación en su enfoque metodológico y el uso sistemático de herramientas de software que integren de manera eficaz, tanto los procedimientos técnicos como administrativos y de carácter contractual, cubriendo todas y cada una de las fases y etapas que involucran el desarrollo de esta área.

Los procesos indicados, que son de naturaleza altamente compleja, en muchos de los casos se los ha aislado con fines administrativos, aspecto que ocasiona pérdida en su concepción integral, manejo y administración, por estar limitada la perspectiva de integración entre los procesos transversales de Gestión Ambiental, indispensables para cumplir con la Normativa Ambiental y exigencias contractuales, y la necesidad de preservar el entorno en todas su facetas, llámense sociales, técnicas, económicas e incluso aquellas de carácter subjetivo que tienen que ver con la percepción que tiene el ciudadano, usuario de estas obras.

Las metodologías de desarrollo y caracterización de procesos a través de las herramientas de software (S. White, D. Miers, 2010), permiten que se integren los componentes de diseño, desarrollo, implementación e implantación de sistemas, mediante parámetros alimentados en los sistemas indicados que responden al conocimiento y experticia del diseñador en el proceso técnico-administrativo, sin preocuparse de la programación del software, la cual está resuelta con herramientas tales como los lenguajes BPEL (Business Process Execution Language) (Fischer Layna Edited 2010), misma que resulta innecesaria en la mayor parte del proceso, y muy restringida en la implementación. Esto permite que el usuario-diseñador se centre en la problemática antes que en la herramienta de trabajo, logrando mayor eficiencia y eficacia en el proceso constructivo. Las interfaces son amigables así como la curva de entrenamiento es muy corta, aspecto que incide sobre la productividad del usuariodiseñador, permitiendo desarrollar su creatividad (De Bono, Edward (2011), antes que centrarse en la herramienta.

Este modelo de diseño responde a los aspectos macro de la gestión, incluido el componente ambiental, concatenando todos y cada uno de los procesos y subprocesos. En la resolución del problema, se emplean enfoques como el de top-down (arriba-abajo) y (bottom-up), a fin de hacer un "barrido" de los procesos involucrados; sin embargo, esta visión piramidal no es suficiente para la resolución del problema, porque no considera aspectos transversales que obedecen a una práctica diaria en la gestión de los proyectos. Con el presente enfoque, además de dar una macro visión del problema, se pretende, en un corto espacio, que el lector tenga una idea esencial de los aspectos inherentes al problema, representados en el modelo tanto en la parte estática, como dinámica y funcional.

El modelo propuesto es un "sketch" de construcción preliminar que establece ideas generales acerca del problema.

Debemos señalar que el principal objetivo en el desarrollo de un sistema de software, es precisamente lograr un diseño preliminar que tenga la consistencia adecuada para la resolución del problema planteado y, un diseño detallado que resuelva de forma granular, todos y cada uno de los aspectos propios de la implementación. En ese sentido, se requiere que el diseñador conozca en profundidad el problema a resolver, a fin de que disponga de todos los elementos micro que van a ser claves para llegar al diseño "sin errores", que satisfaga las necesidades del usuario final.

Debemos recordar que la variabilidad de las herramientas software, así como también todos los dispositivos de hardware, los cuales no están normalizados, son elementos que deben ser seleccionados de una manera rigurosa, con el fin de que no se constituyan en algún momento, dentro de cualquiera de las etapas de diseño y desarrollo, en un obstáculo tecnológico. Se debe apuntar a soluciones probadas en el mercado de las tecnologías de información y comunicaciones, que por su madurez tengan resueltos los innumerables problemas propios del área de ingeniería de sistemas informáticos.

\section{Desarrollo del Problema}

A fin de proceder al desarrollo del problema y previo a plantear el diseño del problema, desde una perspectiva tecnológica, debemos caracterizar las fases o etapas de la Gestión Ambiental, consistentes en:

a) Estudio de Impacto Ambiental

b) Evaluación de Impacto Ambiental

c) Valoración de Impacto Ambiental

d) Declaratoria de Impacto Ambiental

e) Ejecución de Obra

f) Control y Fiscalización

a) Estudio de Impacto Ambiental

Contiene todos los aspectos que deberán ser controlados 
en la etapa de ejecución del proyecto de ingeniería, sobre la base de las definiciones realizadas dentro del estudio; para lo cual, es necesario efectuar un relevamiento de información de las variables que afectan el proyecto. Este esquema metodológico (Gráfico 1), según se menciona en (Jordán, Glenda, 2008), ha sido adaptado y modificado, para el presente trabajo, observándose los aspectos principales de la Investigación y de los resultados del Estudio de Impacto Ambiental. Este estudio, constituye la contrapartida del proyecto de ingeniería, por cuanto es el componente principal y objetivo que permitirá en la etapa de ejecución, efectuar los controles, valoraciones y correcciones necesarias para lograr que se cumplan las normas y disposiciones legales de manera orgánica, es decir, que no sean "letra muerta", en el momento de desarrollar los proyectos.

Con estas reflexiones, describimos mediante el siguiente diagrama una propuesta de diseño, conforme la herramienta y metodología adoptada.

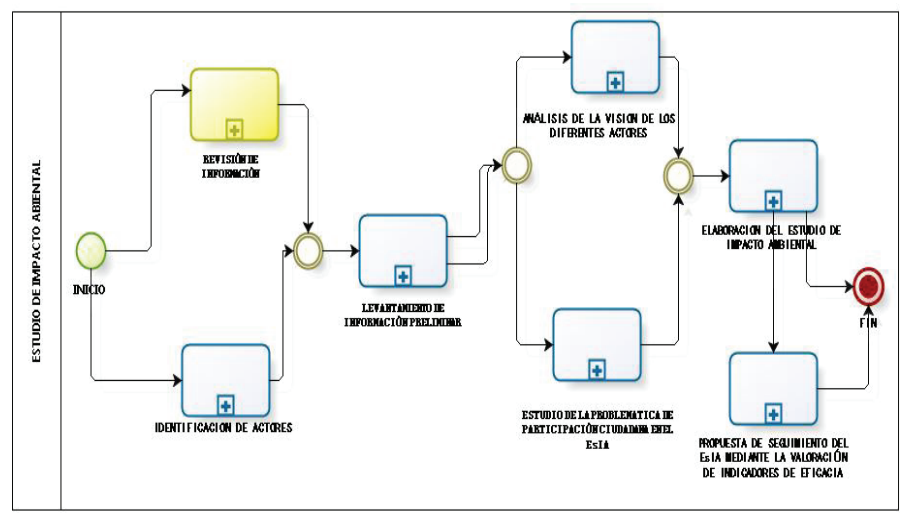

Gráfico 1 observamos como punto de partida el evento "Inicio". De hecho, todo proyecto tiene este elemento, que es un hito para la herramienta.

La siguiente etapa de análisis es la revisión de información. Este módulo es indispensable para determinar cuáles son los elementos y estructuras base que delimitan el proyecto de ingeniería, así como también la normatividad existente, aplicable al proyecto. No se quiere decir que la normativa va a resolver el problema de implementación, pero se entiende que los especialistas de ingeniería, tendrán que adecuar sus diseños a los requerimientos que impone la normatividad. Aquí debemos crear un paralelismo y transversalidad con el proyecto de ingeniería, para que las actividades o rubros de construcción propios de la obra, tengan de forma simultánea y previa a su ejecución, el aval de los especialistas en Ingeniería Ambiental aplicada al campo de la vialidad. Debemos señalar que en los proyectos de Vialidad, hay que diferenciar las obras viales (vías), de las estructuras mayores (puentes), cuyo tratamiento debe darse de forma diferente, por cuanto intervienen factores distintos de la obra vial (por ejemplo: aspectos hidrológicos, estructurales, etc.) MTOP (2013), Manual_NEVI-12_VOLUMEN_2.

Se identificará, paralelamente, a los actores intervinientes en la realización del proyecto, tanto del área de ingeniería como de las áreas ambientales. En el sistema, estos actores se encuentran debidamente identificados por competencias.

Es preciso recalcar que en la metodología de gestión por procesos, los actores no corresponden a personas sino a funciones.

En cuanto al relevamiento de información, en la etapa de investigación de campo, se deberá hacer el levantamiento de información necesaria y suficiente para la resolución macro del problema. En este sentido, se hará uso de diferentes esquemas metodológicos, destacándose entre estos principalmente: la entrevista, el cuestionario y la encuesta. Con estos métodos de trabajo, es posible obtener la información adecuada para elaborar los diseños del sistema. En la parte formal, se aplicarán buenas prácticas de desarrollo, conforme a estándares internacionales, generalmente aceptados.

Ejecutado el relevamiento, y con el fin de tener información gerencial útil para la modelización, se investigará cuál es la visión de los diferentes actores, qué es lo que se espera del modelo y finalmente del sistema implementado. En este aspecto se deberían clarificar los contenidos y alcance del sistema, es decir los elementos de contorno y especificaciones para lograr una apropiada implementación.

En el análisis de la problemática de participación ciudadana, se efectuarán los acercamientos, conforme a las metodologías inherentes al Estudio de Impacto Ambiental, manifestando a los grupos afectados y, en especial a sus representantes ¿cuáles son las ventajas de implementar los proyectos y de qué forma se harán el monitoreo y seguimiento?

Para el efecto, se establecerán estrategias de gestión que conlleven a suscribir acuerdos mutuos de participación, elementos que están reflejados en el sistema de software y los componentes contractuales del Proyecto. Para el análisis y desarrollo del Estudio de Impacto Ambiental, hay que tomar en consideración las metodologías, generalmente aceptadas en esta área, hasta la consecución del documento final, en el cual debe instrumentalizarse la mecánica y acciones a seguir, paralelamente a la construcción de la obra vial. Es necesario que todos los actores del proceso conozcan en profundidad el contenido del Estudio de Impacto Ambiental, a fin de tener una base 
mínima de acuerdos entre los actores (MTOP (2013), Manual_NEVI-12_VOLUMEN_2A). El enlace será el Modelo Sistematizado del Proyecto de Ingeniería y el Estudio de Impacto Ambiental, para cumplir con las metas planteadas, evitando los conflictos al interno del proyecto o con la sociedad.

A fin de elaborar los indicadores de eficacia, se basará en el Estudio de Impacto Ambiental, en la parte atinente al manejo de cronogramas técnico y económico y redes del proyecto, elementos que permitirán controlar y medir el avance de las actividades, tanto del Proyecto de Ingeniería, como del Estudio de Impacto Ambiental y los niveles de desempeño. Es posible, en esta parte, implementar sistemas de Tablero de Comando (Balance scorecard) que acompañen al Proyecto y disparen las alarmas necesarias en caso de producirse retrasos o distorsiones en la ejecución del Proyecto.

Como producto resultante, de manera formal, tenemos el Estudio de Impacto Ambiental (Es|A), que será el documento base para la Supervisión, Fiscalización, Control y en etapa futura, la Auditoría Ambiental.

El EsIA es el elemento de trabajo que dinámicamente nos dará información cuali-cuantitativa en todas las fases de ejecución del contrato, y por tanto también deberá reflejar los cambios, modificaciones o agregaciones que se hagan al proyecto.

La dinamia del EsIA, es la resultante de los cambios al Proyecto de Ingeniería. En tal sentido, se documentará, de manera exhaustiva, todas las acciones y modificaciones producidas en el contrato y en los componentes de la gestión ambiental.

\section{b) Evaluación de Impacto Ambiental}

Se requiere una valoración conforme a los requerimientos metodológicos, optando una metodología o método adecuado para su implementación. Para ilustrar lo indicado, se ha construido el esquema siguiente (Gráfico 2), que guarda una relación de continuidad con la etapa de Estudio de Impacto Ambiental. Aquí se observan tres componentes principales del análisis, que se refieren a la revisión del Proyecto de Ingeniería. Es necesario señalar que, en este punto, confluyen los requerimientos expuestos en el Estudio de Impacto Ambiental, con las actividades de Ingeniería propias de la especialidad. Además, se debe conjugar aspectos de carácter administrativo, legal y económico, acordes a la problemática planteada. La flecha con línea entrecortada, establece un enlace con la Etapa de Valoración de Impacto Ambiental.
Reconocemos una fase preliminar que es el análisis múltiple del proyecto de ingeniería. Para esto, los actores intervinientes, deberán establecer claramente ¿cuáles son las tecnologías a aplicar, los procesos y procedimientos necesarios en la parte ambiental, que permitan controlar el desarrollo del proyecto?

En los procedimientos jurídico-administrativos, es fundamental que las partes (contratista y contratante), definan el marco de referencia del contrato y de esa manera se precise de forma específica cada fase y etapa de construcción y/o mantenimiento vial. Los rubros de construcción se normalizan a través de la aplicación de las Leyes y Reglamentos inherentes a la materia.

El procedimiento técnico-ambiental, considerará las etapas y actividades del proyecto de ingeniería y las técnicas de control adecuadas para que se desarrolle dentro de los estándares de calidad. Se sugiere disponer de un Sistema de Gestión Ambiental (Norma ISO 14001), tanto en la empresa como en las organizaciones intervinientes en el Contrato. El fiscalizador deberá inteligenciarse acerca de los procedimientos a implementar conforme la normativa aplicable.

Para el procedimiento económico-financiero, se dispondrá de los cronogramas valorados del proyecto de ingeniería y del Estudio de Impacto Ambiental, que permitan controlar los recursos, su disponibilidad y la gestión adecuada para que la obra de ingeniería no sufra retrasos que afecten a la administración de los recursos y que en consecuencia, originen pasivos ambientales.

Deben evitarse los retrasos en la ejecución del Proyecto, que en lo económico afectan a ambas partes, originando incrementos innecesarios de costos que luego se verán reflejados en la modificación de las Fórmulas de Reajuste de Precios, y por ende en el valor de las planillas a ser pagadas a los contratistas, en desmedro de la economía del país. El lucro cesante aquí producido tendrá impacto en la no realización de otros proyectos de Ingeniería, que generalmente también son emergentes y de alta necesidad para la comunidad. La cautela con la que el Administrador del Contrato o Gerente deba llevar a cabo el Proyecto, garantizará que no se produzca este tipo de pérdidas que finalmente deberán incorporarse como gastos.

A continuación, identificamos los impactos que deben registrarse en el sistema para monitorear en la etapa de producción de los sistemas. Esta identificación es propia para cada proyecto de ingeniería, por las condiciones disímiles, siendo cada proyecto específico. 
Para la predicción de Impactos, se establecerán los escenarios adecuados a fin de observar las posibles situaciones que pueden presentar en el desarrollo del proyecto de ingeniería. La predicción de Impactos deberá ser el resultado del análisis cuali-cuantitativo y del estudio del espacio de probabilidades en el que se analicen. Podemos tener aquí un sinnúmero de alternativas, pero deberemos ayudarnos de las experiencias $y$, en definitiva, de nuestras heurísticas sobre el manejo del problema.

Finalmente, será necesario documentar los impactos identificados y resueltos en el proceso de Evaluación, lo cual nos entregará cuadernos de trabajo para el seguimiento de la obra.

Es preciso señalar aquí que el documento resulta nominativo, una vez que refleja situaciones puntuales del proceso, y por tanto tendrá que ser actualizado y/ o revisado para adecuarse a las múltiples y nuevas situaciones que se presentan, a diario, en la ejecución de los proyectos de ingeniería.

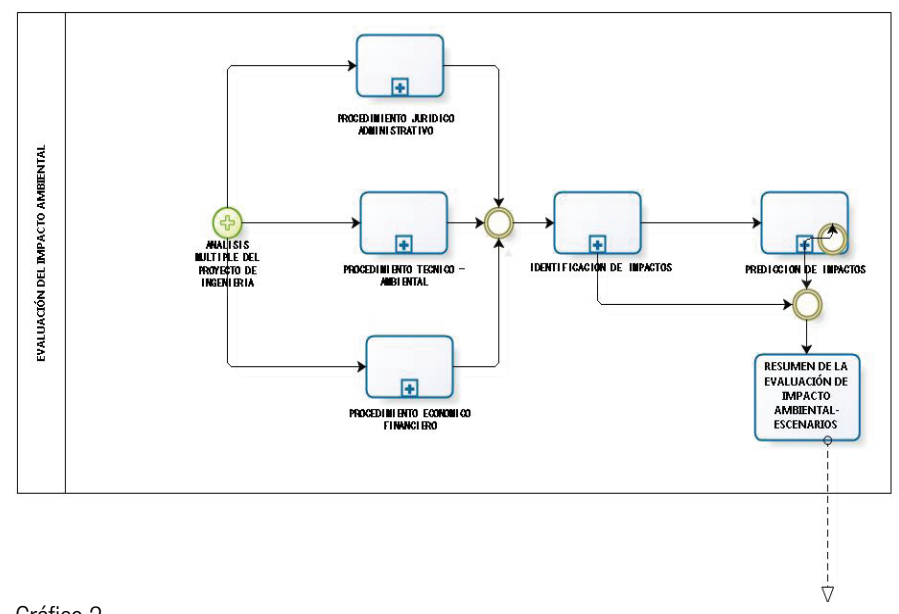

Gráfico 2

\section{c) Valoración de Impacto Ambiental}

Esta fase última del estudio, comprende la estructuración y valoración cuali-cuantitativa, generándose matrices como Leopold y otros autores, para determinar la significancia y grado de afectación de las actividades del proyecto de Ingeniería.

Cuando el proyecto ha sido aprobado, se valoran los Impactos que pueden darse en el proyecto, y en caso de detectarlos, se procede a definir qué tipo de controles debemos efectuar. Usamos las metodologías propias de Gestión Ambiental. Una vez efectuada esta actividad, construimos las matrices de valoración ambiental.

Para la elaboración del componente Valoración de Impacto Ambiental, entendemos que la misma se la debe hacer durante la ejecución del proyecto, dinamizando las propuestas y estructuras nominales (matrices), extrapoladas a las realmente ejecutadas.

Si hacemos en este punto una abstracción, debemos señalar que la valoración preliminar podría cambiarse durante el proceso de ejecución mismo. Esta "dinamia entrópica del sistema" no deberá afectar a los objetivos del control, pero si permitirá tener nuevos esquemas, mejor afinados, dentro de la ejecución de los proyectos, hasta llegar a un ciclo de estabilización adecuado para el proyecto ejecutado y el cumplimiento del componente ambiental.

Debemos tener presente que, en la ejecución de un proyecto, el componente variabilidad, no es fácilmente controlable y, la única forma de reducir su efecto es disponer de los elementos y estudios previos (por ejemplo: Estudios de suelos, Geotecnia de suelos, etc.), elementos constituyentes esenciales del proyecto, y que garantizarán su óptima ejecución.

Sin embargo, y muchas ocasiones en la realidad, que por cuestiones de "emergencia" real o imaginaria se soslaya este tipo de análisis, dando como resultado que los proyectos y su componente ambiental, fracasen por la impensada realización y la superficialidad con la que se abordan estos estudios, o simplemente no se los hace.

Resulta pertinente aplicar modelos de simulación de la administración del medio ambiente, en forma de matrices, incluyendo los criterios necesarios que permiten discriminar los aspectos principales de los accesorios, estableciendo varios escenarios dentro de la investigación (Boland et. Al., 2001).

En la propuesta actual, todos estos factores se "documentan", siendo en lo posterior la clave principal para procesos externos de Auditoría Ambiental necesarios para juzgar la calidad, costo y oportunidad de las medidas ambientales y de índole similar adoptadas.

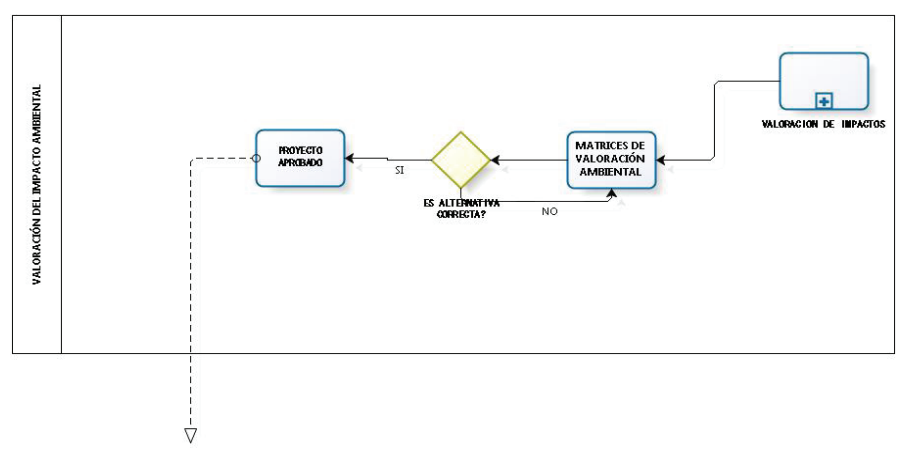

Gráfico 3 
Revisado el diagrama anterior, observamos un enlace que se produce con la etapa de Declaratoria Ambiental.

\section{d) Declaratoria Ambiental}

Resume y gestiona la aprobación o no del proyecto de Ingeniería, sobre la base de las consideraciones socioambientales del mismo. Se genera un documento de declaratoria.

Para el efecto, el proyecto de ingeniería y los Estudios de Impacto Ambiental se someten al análisis de expertos en la materia y luego a un proceso de alegación pública. De esta forma, el proyecto y su contrapartida (Estudio de Impacto Ambiental), son analizados en una perspectiva distinta de la técnica, involucrando temas de carácter sociológico, psicológico, urbanístico, de movilidad de personas, zonificación por reubicación vial, compensaciones económicas, litigios.

En consideración a esta problemática y luego de ejecutadas estas actividades, se procede a emitir una resolución administrativa (por ejemplo, Acuerdos Ministeriales), en la que se aprueba la realización de la obra. En caso de que no sea procedente, 0 exista alguna limitante para la realización del proyecto, se archiva el proyecto o se deja pendiente su ejecución sobre la base de las prioridades establecidas en la Programación de Obras (Plan Operativo Anual, Plan Anual de Inversiones, Plan Plurianual, Plan Estratégico de Desarrollo Institucional, etc.), elementos clave de la administración.

Una vez que se aprueba el proyecto, se pasa a la siguiente etapa que es la ejecución de obra.

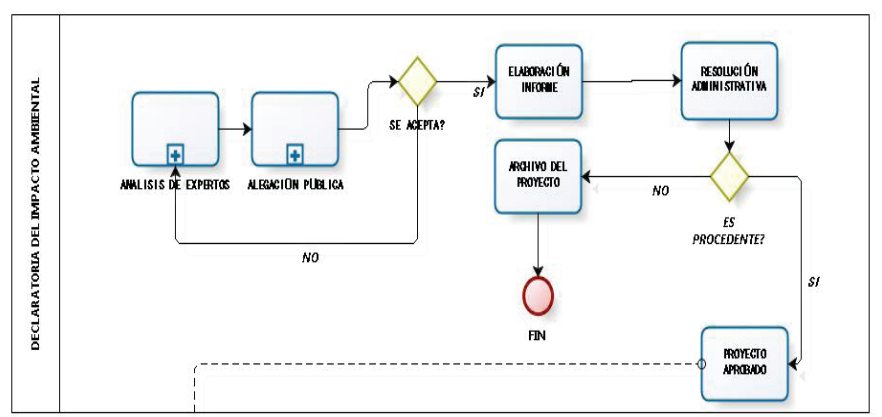

Gráfico 4

\section{e) Ejecución de la obra}

Esta Etapa es la más crítica del proyecto, por cuanto se realizará el seguimiento a las diferentes actividades y tareas constructivas, partiendo de los diseños preliminares y definitivos, hasta la verificación de las tareas registradas en el "libro de obra", que es el instrumento técnico que incorpora los aspectos micro de la construcción. Es necesario señalar que en esta Etapa, ha de efectuarse el acompañamiento permanente con los responsables de la Auditoría de Gestión Ambiental, mediante la verificación del cumplimiento de los Indicadores de Eficacia, parámetros planteados en la primera fase, así como también el trabajo de validación del Fiscalizador de la Obra, en función de los rubros fijados dentro del proyecto, y que corresponden al componente de Gestión Ambiental. Por separado, expondrán los criterios técnicos y particularmente el cumplimiento de las especificaciones, reglas y recomendaciones fijadas en el Estudio de Impacto Ambiental; entendiéndose que hay una responsabilidad compartida entre los dos actores del proceso. Para el control de los rubros, tanto de construcción, así como de manejo ambiental, se considerará lo dispuesto en la normativa general existente sobre la materia.

La autoridad competente, decide la ejecución de la obra de ingeniería e invita a los promitentes contratistas a participar en la ejecución de la obra. Se llama a concurso, en las modalidades previstas en la Ley, y en caso de que las ofertas sean favorables, se califica y selecciona la mejor oferta, técnica y económica. Realizado esto, se inicia la ejecución, ejerciendo los controles y seguimiento al mismo tiempo. Simultáneamente se verifica el cumplimiento de lo estipulado en el Estudio de Impacto Ambiental, mediante la incorporación de indicadores de eficacia. Si los indicadores son adecuados, se continúa con la ejecución de la obra. En caso contrario se ejecutará obras o actividades de mitigación, a fin de cumplir con los rubros previstos en el contrato y el Estudio de Impacto Ambiental.

Si esta actividad no es suficiente, el Fiscalizador se notificará al contratante a través de los mecanismos y recursos existentes (Sistema BPM), y que luego conocerá el Supervisor y /o el Administrador del Contrato.

En caso de existir correcciones, se procederá a emitir órdenes de trabajo para paliar los efectos que la actividad o rubro han ocasionado. Si el contratista no acepta, la Autoridad competente adoptará las acciones legales para corregir esta problemática planteada. Para sistematizar adecuadamente, el aplicativo integrará un Sistema de Soporte de Decisiones apoyando la Gestión Gerencial. 


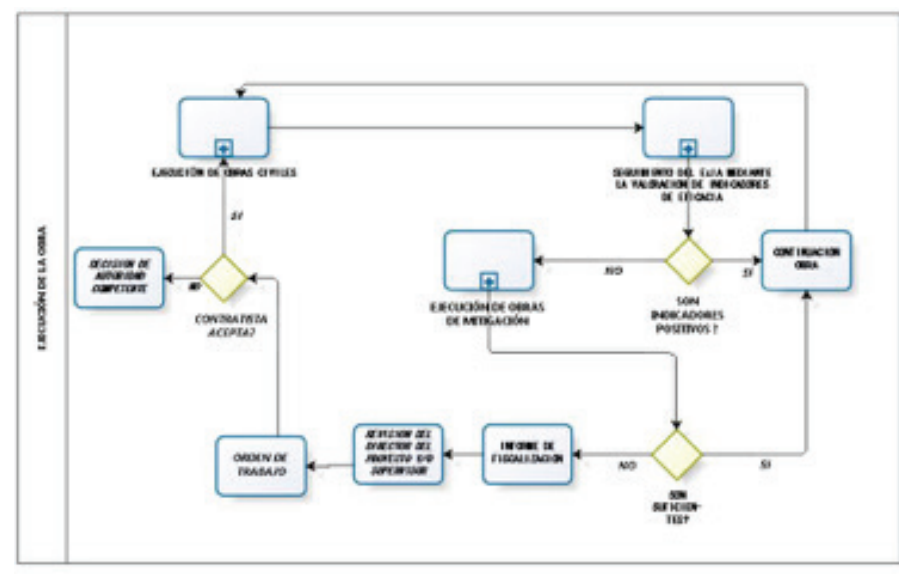

Gráfico 5

\section{f) Control y Fiscalización:}

Previo a realizar este análisis, graficamos el diagrama correspondiente, a fin de visualizar las interrelaciones existentes en estos procesos.

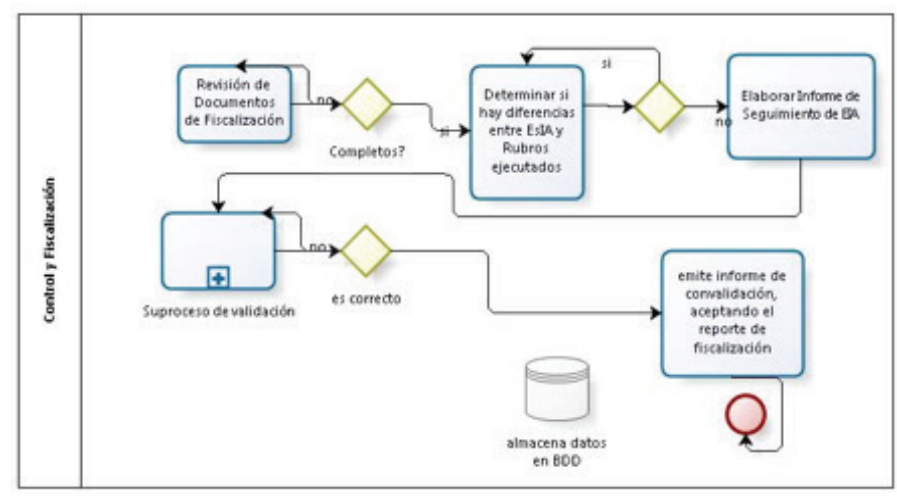

Gráfico 6

Una vez que el Inspector Ambiental recibe los documentos enviados por la Fiscalización, procede a validar la información contenida, y a determinar si efectivamente contenida, y a determinar si efectivamente se ha cumplido la ejecución de rubros ambientales, y los de ingeniería que deben estar descritos en la normativa. Si se ha cumplido esta actividad a cabalidad, determina si ha habido diferencias entre los rubros señalados en el EsIA y lo que efectivamente se ha ejecutado. En caso de haberse producido diferencias, solicitará los justificativos técnicos y ambientales para aprobar estos rubros. Si se han cumplido, se deberá acatar lo que establece el procedimiento anterior; es decir que el Inspector Ambiental, procederá a establecer los correctivos y, eventualmente señalará las acciones que deba cumplir el Fiscalizador. Si no se han producido cambios o desviaciones que sean críticas, deberá elaborar el informe de seguimiento que corresponde a la Evaluación de Impacto Ambiental. Se someterá a un subproceso de validación, que es un proceso exhaustivo del cumplimiento o no de las acciones establecidas en el EsIA y otros documentos de apoyo.
Concluida esta actividad, emitirá el informe de convalidación, aceptando en todo 0 en parte lo ejecutado. Esta información se almacenará en la base de datos por ser necesaria para el momento en que se produzca la liquidación del contrato, por cualquiera de las causales establecidas en la Ley. La información así procesada, y dadas las características del software, podrá ser almacenada en la "nube" (cloud), una vez que está habilitada esta funcionalidad dentro del paquete de software. En este caso, debe cuidarse que existan respaldos en varios sitios de la información almacenada a fin de evitar inconvenientes futuros por eventual pérdida de información. Los datos y resultados tendrán que ser "congelados", y guardarán estrecha relación con los informes que se genere en la Inspección Ambiental.

Debemos señalar que este proceso será finalmente escrutado por la Auditoría Ambiental, en donde se establecerá si existen responsabilidades por acción u omisión de los ejecutores del contrato.

Las penalizaciones a que dieren lugar estas acciones $u$ omisiones, son extensivas a todos y cada uno los personajes intervinientes en la ejecución y/o control de la obra de ingeniería.

\section{Resultados y discusión}

Se puede observar en el presente trabajo los siguientes resultados:

Para caracterizar el modelo lógico, se ha procedido a realizar un levantamiento de información, acerca de los procesos y subprocesos inherentes a la problemática propuesta. Este relevamiento es consistente con la normativa aprobada y que debe ser analizada en detalle, a fin de establecer las condiciones de contratación y los aspectos operativos necesarios para cumplir con la obra, objeto del estudio.

\section{a) Desarrollo del Modelo Físico}

En el modelo, se han establecido varios "carriles" o pool, que nos permiten, dentro de la metodología y herramienta empleadas, definir las diferentes etapas del proceso, hasta llegar a un alto grado de atomización. Esta normalización es fundamental al momento en que se pase a la siguiente etapa de diseño funcional, en la cual deben interactuar componentes y elementos internos 0 externos al proceso, generando una dinámica en las entradas y salidas de los procesos y subprocesos. El modelo exige una retroalimentación y afinación del mismo, a fin de que exista 
una correspondencia entre los componentes del modelo. La herramienta permite depurar en la parte sintáctica, a fin de que no se produzcan inconsistencias en el proceso, actividad 0 etapa subsiguiente. Esta facilidad, así como la generación de documentación en forma automática, hacen posible desarrollar en forma rápida y eficaz, tanto el modelo de procesos, modelo funcional y el modelo de datos, para conformar la base de información necesaria en la etapa operativa.

Para el desarrollo del modelo físico y con el fin de que la implementación se automatice, el arquitecto de aplicaciones, deberá emplear la misma herramienta en su versión completa (P.ej. Bizagi Studio), la cual integra todos los aspectos de desarrollo de software. El desarrollador, conocerá a fondo los procesos inherentes a la aplicación misma, a fin de llegar a la consecución de un producto perfeccionado y paralelamente integrado.

El uso de la herramienta propia de diseño y desarrollo, evitará la migración desde otros sistemas informáticos, lo que acarrea, necesariamente, que deba ajustarse mediante herramientas de programación convencionales, asunto que resulta inoficioso y de gran impacto económico en la implementación.

\section{b) Implementación del Modelo Físico con la Herramienta BPM}

Las herramientas de desarrollo de procesos, se asemejan de alguna forma a las herramientas de desarrollo rápido de aplicaciones, con la diferencia de que las primeras pueden ser manipuladas y gestionadas por directivos

y técnicos con una formación reducida en sistemas computacionales a nivel técnico, pero que tienen las habilidades de gestión que les permita entender el negocio en forma multilateral y multidisciplinaria, mientras que las segundas, obligatoriamente deben ser manipuladas y gestionadas por especialistas en Informática, creándose una dependencia profunda entre el gestor y el informático, la cual para efectos prácticos no es adecuada por la rapidez con que se ejecutan los procesos y actividades

Para lograr este objetivo, es preciso entonces utilizar herramientas integradoras, donde se produzca invisibilidad del software de base, únicamente apareciendo elementos necesarios en la parametrización de los sistemas.

Efectuada esta parametrización, se procede a la revisión de los componentes del "backend" (base de datos), la misma que debe ser de elección cerrada para lograr la optimización de los recursos. En esta parte se limita a motores de bases de datos comerciales que cumplen estándares de calidad. No está disponible para todos los motores y versiones de bases de datos.

Instalado el motor de base de datos, se procede a implementar la base y efectuar el afinamiento correspondiente para lograr el desempeño adecuado del sistema. Aquí se debe precisar que las bases de datos provistas por la industria, tienen la madurez y complejidad necesaria, que simplifica las tareas del administrador de base de datos.

El concepto de "carrusel" que maneja la herramienta, permite regresar a etapas previas para hacer los cambios 0 ajustes, afinando la resolución del problema sin afectar los contenidos existentes en el "backend", Iogrando resolver de este modo uno de los problemas inherentes a la Ingeniería del Software que es la variabilidad. Esta variabilidad representa los cambios entrópicos del sistema y su posible desestabilización. El diseñador deberá tener el cuidado especial, en proponer procesos y subprocesos que estén debidamente avalados y que sean sencillos de demostrar.

Como estrategia para el desarrollo, se mapeará los procesos y subprocesos, y someterlos a pruebas críticas para garantizar que no contengan errores de ninguna clase. El diseñador profundizará el análisis del proceso y los sistemas que resuelven operativamente la problemática.

Adicionalmente, como buenas prácticas de desarrollo de software, utilizando las mismas herramientas del paquete, se procederá a documentar los procesos y subprocesos, hasta conseguir el producto final deseado.

Nótese que, al existir una documentación explícita, el sistema puede ser intervenido por otra persona distinta del diseñador original, sin perder significación lo que ha sido desarrollado y/ o almacenado.

Finalmente, y dentro de este punto, la capacitación en la aplicación obtenida, y el entrenamiento para el personal, constituirán factores críticos de éxito en la implantación de los sistemas y procesos.

c) Análisis de correspondencia de los componentes constructivos y ambientales

A través del uso de la herramienta BPM, podemos visualizar las diferentes interrelaciones entre el proceso constructivo y el proceso de gestión ambiental, relaciones que nos permiten realimentar información desde y hacia cada sistema. 
Esta sinergia entre las dos visiones, se observa en los gráficos 1, 2, 3, 4 y particularmente 5. Se considerará además que los cambios que se apliquen a los procesos, por modificatorias en la legislación y / o los términos contractuales del proyecto, tienen que estar reflejados en el proceso. La entropía de estos sistemas es alta, en tanto existe variabilidad estructural en el componente jurídicoadministrativo, aspecto que incide necesariamente en la estabilidad del sistema. Sin embargo, las herramientas BPM se caracterizan por ser altamente adaptivas, y esta flexibilidad hace que puedan acoplarse a nuevos procesos y subprocesos, 0 en su defecto se eliminen 0 modifiquen otros, tanto en la estructura como en la funcionalidad sin que afecten a la estabilidad del sistema y sus aplicaciones.

La importancia de usar sistemas BPM, es la facilidad con que se pueden estructurar procesos, y la generación de aplicaciones o su conexión, reduciendo de manera significativa los procesos de programación, aspecto que redunda necesariamente en la posibilidad de rehacer los módulos construidos, eliminarlos o simplemente volver a crearlos. Los tiempos de desarrollo se simplifican ostensiblemente, por cuanto se han desarrollado dentro de la herramienta, elementos estandarizados que permiten un desarrollo rápido de las aplicaciones.

Así concebidos los procesos, es fácil notar que la sinergia anteriormente mencionada es posible, precisamente por la facilidad estructural y los aspectos conceptuales ya implementados. En tal virtud, lo que se hace internamente en el sistema, es incorporar objetos los cuales son invocados de manera selectiva, en base a la correcta utilización de los elementos gráficos (símbolos) de BPM.

En cuanto a los sistemas subyacentes, necesariamente operacionales, pueden ser adaptados o acoplados a la estructura principal, sin que se afecte la funcionalidad de los sistemas indicados. (Braja M. Das, 2001), (Torres Milton, 2010).

Una vez que se implementa la aplicación, ésta se convierte en una poderosa herramienta, como un valioso elemento para el entrenamiento de personal, al organizar de manera precisa todos y cada uno de los procesos intervinientes en la Gestión, tanto a nivel de Ingeniería como Ambientales.

Es necesario recalcar lo propuesto en MTOP (Manual_ NEVI-12_VOLUMEN_4, 2013), acerca de los convenios relevantes para el cumplimiento de las normas ambientales. También es fundamental, considerar el Patrimonio Natural y reservas declaradas, para un control adecuado de los proyectos.

\section{Discusión del Modelo Propuesto}

De lo observado, podemos decir que el sistema propuesto puede ser una alternativa de solución viable a la problemática socio ambiental generada por los proyectos viales.

El modelo constructivo plantea la necesidad de un trabajo de carácter multidisciplinario que, de forma concurrente aporte a las soluciones técnicas, económicas y particularmente ambientales que el proyecto requiere (Fischer Layna, Edited 2010).

La implementación es transparente a la herramienta, una vez que se emplean elementos normalizados, cuya significación está definida adecuadamente.

En el desarrollo del problema, observamos varios aspectos importantes que deben ser mencionados en este acápite. Estos son:

- Construcción horizontal y vertical del modelo

- Transversalidad en el diseño de los procesos.

- Identificación adecuada de los procesos, incluso de aquellos. que generalmente pasan desapercibidos para los usuarios.

- Sencillez en el lenguaje. Nos centramos en el modelo, no en la herramienta.

- Objetivación de procesos ocultos o que no tienen definición conceptual apropiada. Traslapar.

- Libertad para el manejo del diseño. Se debe prever los traslapamientos de símbolos, lo que podría ocasionar confusión para entender el problema.

- Tener una lista de revisión a nivel de procesos y subprocesos, actividades y tareas.

Al plantear esta metodología, se propone: facilitar la integración entre modelos, sistemas, actores y usuarios, de tal forma que responda a las necesidades, tanto del punto de vista tecnológico, así como del ambiental. En cuanto a los tiempos de implementación, se debe considerar un mínimo de seis meses y un máximo de doce meses, ya que una extensión a esta duración, puede significar un incremento de costos y cambios en el enfoque de desarrollo de sistemas.

\section{Conclusiones}

- La Metodología propuesta es totalmente adaptable a 
Ios fines propuestos, por cuanto permite establecer una sinergia entre los dos componentes, tanto del Proyecto ingenieril, así como del Ambiental.

- La herramienta BPM, con todos los elementos y componentes, presenta facilidades para conceptualizar los problemas multidisciplinares.

- En el presente trabajo, el uso de esta herramienta con un enfoque técnico, da una visión diferente de su habitual empleo en aplicaciones de carácter comercial, 0 similares.

- Los aspectos técnicos, en elárea de Ingeniería, se pueden acoplar a las condiciones propias del proyecto, sin que esto afecte el carácter general de la implementación.

- Los aspectos de complejidad accidental, se ven reducidos por la versatilidad de la metodología propuesta, y la herramienta de software aplicada.

- La conformidad del modelo con los elementos prácticos, se comprueba a través de la funcionalidad del modelo.

- La construcción de los modelos, adicionalmente a los aspectos teóricos y metodológicos, es afectada necesariamente por la experticia del diseñador en las dos áreas de especialidad.

- No es factible según el "estado del arte", Iograr la solución única al problema. Esta variabilidad estructural puede limitarse, desarrollando mapas de procesos de carácter general, que a la vez puedan adaptarse a las condiciones propias del proyecto.

- La invisibilidad del modelo, en cuanto a las herramientas de desarrollo se obtiene por la independencia en las capas superiores del diseño de este modelo, en relación a la programación de las herramientas.

\section{Referencias bibliográficas}

S. White, D. Miers (2010), Guía de Referencia y Modelado BPMN, Comprendiendo y Utilizando BPMN, Future Strategies Inc. Book Division, ISBN 13: 978-09819870-3-3, pp. 35

Fischer Layna Edited. (2010), BPM and Workflow Handbook, Spotlight on Business Intelligence, Future Strategies Inc. Book Division, ISBN 978-0-9819870, pp. 147-155.

De Bono, Edward (2011), ¡Piensa! Antes de que sea demasiado tarde, Edit. ESPASA, Trad., pp. 36.
Jordán, Glenda (2008), Gestión Socio Ambiental en la Evaluación de Proyectos Viales en el Perú, Flacso, Tesis de Maestría, pp. 1

Braja M. Das, (2010), Geotecnia en Obras de Infraestructura de Transporte, Edit. MTOP, Quito, pp. 134-135. 136-137 Torres Milton (2010), Pavimentos de Carreteras, Edit. MTOP, Quito, pp. 10-70

Fischer Layna Edited. (2010), BPM and Workflow eBook Series, Methods, Concepts, Case Studies and Standards in Business Process Management and Workflow, Future Strategies Inc. Book Division, ISBN 13: 978-0-9198704-0, pp.12-19

MTOP (2013), Norma ecuatoriana vial NEVI-12MTOP, consultado el 21 de febrero de 2016, http:// www.obraspublicas.gob.ec/wp-content/uploads/ downloads/2013/12/01-12-2013_Manual_NEVI-12_ VOLUMEN_4.pdf; págs. 28-29; 106-107

MTOP (2013), Norma Ecuatoriana Vial NEVI-12MTOP, consultado el 21 de febrero de 2016, http:// www. obraspublicas.gob.ec/wp-content/uploads/ downloads/2013/12/01-12-2013_Manual_NEVI-12_ COMPLEMENTARI0-2.pdf

Boland, R. (2001), Capacitación en Administración del Medio Ambiente, Vol. IV, Simulación de la Administración del Medio Ambiente, Programa: OIT/PNUMA, Ginebra, Edit. Alfaomega

http://www.omg.org/spec/BPMN/2.0.2/PDF/ FORMAL-13-12-09. Consultado el 26 de diciembre de 2016 